\title{
Lebenslang trotz schwerer psychischer Störung - Abkehr von der restriktiven Auslegung der Mordmerkmale?
}

\author{
Männerfleisch als Fetisch: Mord, Tötung auf Verlangen oder Totschlag. Was ist die an- \\ gemessene Strafe für den Kannibalen von Rotenburg? Diese Antwort dürfte noch nicht \\ gefunden sein.
}

Gönke Jacobsen

\begin{abstract}
$\int$ as Landgerichtes Frankfurt a. M. hat unter Vorsitz von Richter Klaus Drescher am 9. Mai 2006 den Angeklagten, für den sich die Bezeichnung als »Kannibale von Rotenburg « etabliert hat, erneut für das Töten, Zerteilen und Verspeisen seines Opfers verurteilt, und zwar wegen Mordes zu lebenslanger Freiheitsstrafe. Zuvor wurde das Urteil des Landgerichts Kassel, das acht Jahre und sechs Monate Freiheitsstrafe wegen Totschlags vorsah, durch den BGH aufgehoben (BGH Urt. v. 22.4.2005, NJW 2005, 1876 ff.). Der Fall des sog. Kannibalen zeigt nicht nur das große menschliche Bedürfnis nach abartigen Sensationen, dem die Berichterstattung in der auch internationalen Presse gerne nachkommt - der »Kannibalenplot « wurde schon verfilmt, aber wegen einer einstweiligen Verfügung nicht veröffentlicht -, sondern auch welcher Symbolwert der lebenslangen Freiheitsstrafe zugemessen wird.
\end{abstract}

In NK 2 - 2004 wurde die Zunahme der wegen Mordes zu lebenslanger Freiheitsstrafe Verurteilten dargestellt und die Gründe für diese Rechtspraxis zur Diskussion gestellt (Morgenstern NK 2/2004, S. 52 ff. und Jacobsen S. 56 f.). Morgenstern zeigte, dass sich in absoluten Zahlen die entsprechenden Verurteiltenzahlen der lebenslangen Freiheitsstrafe seit den 90er Jahren auf 136 im Jahr 2001 gut verdoppelt haben, was aus ihrer Sicht auf eine restriktivere Sanktionspraxis zurückzuführen sei, zumal genau in dieser Zeit die Maßregel der Sicherungsverwahrung ausgebaut worden ist. Deutschland sei weit davon entfernt den Gebrauch der lebenslangen Freiheitsstrafe bzw. Verwahrung einzuschränken. Zugespitzt kann diese Entwicklung auch als Dramatisierungsdruck angesehen werden, den Politik und Medien auf die Rechtsprechung ausüben (näher Jacobsen). Dieser Druck könnte aber, so die vorsichtigere Deutung von Jacobsen, auch einer tatsächlichen Veränderung geschuldet sein, auch eine neue Qualität der Brutalität der Tatdurchführung und der "gefühlte Eindruck einer zunehmend verrohenden Gesellschaft könnten das veränderte Sanktionsverhalten erklären.
Das erste Instanzgericht, das Landgericht Kassel, war im Januar 2004 noch bemüht trotz des spektakulären Falls eine moderate Bewertung vorzunehmen und dem Plädoyer der Staatsanwaltschaft auf Mord nicht zu folgen. Dies wurde von Jacobsen als bemühte Auseinandersetzung mit der restriktiven Auslegung der Mordmerkmale und damit als zurückhaltender Umgang mit der Verhängung der lebenslangen Freiheitsstrafe eingestuft (NK 2/2004, S. 57). Die Praxis der Rechsprechung bei der Auslegung der Mordmerkmale erschien damals trittsicher zu sein. Lebenslange Freiheitsstrafe wurde nur dann als angemessene Sanktion aufgefasst, wenn unerträgliches Unrecht geschehen war. Mit dem Urteil des BGH zum Kannibalenfall, dessen Wertungen zu den Mordmerkmalen vom Landgericht Frankfurt a. M. schlicht übernommen wurden, gibt es eine spektakuläre Entscheidung, die diesen Trend umkehrt. Die Ausführungen des BGH sind in dieser Begründung nicht mehr von der Vorstellung geleitet, den Vorgaben für eine restriktive Auslegung der Mordmerkmale zu genügen, sondern stattdessen den Tathergang strikt unter die Mordmerkmale zu subsumieren. In juristischen Besprechungen des Urteils wird hervorgehoben, dass das Merkmal »zur Befriedigung des Geschlechtstriebs « gezielt ausgeweitet wird. Selbst wenn ein Täter bei der Tat kalt bleibt, soll das Mordmerkmal gegeben sein, wenn er sich ein Video anfertigt, um dieses nach der Tat zum Zweck der sexuellen Befriedigung anzusehen (Schiemann, NJW 2005, S. 2350; Kudlich, JR 2005, S. 343; Otto, JZ 2005, S. 799). Selbst der Bundesgerichtshof scheint somit nicht immun zu sein gegen die genannten Dramatisierungstendenzen. Kudlich spricht provokant von einem »fragwürdigen paternalistischen Einschlag« der Bundesgerichtsentscheidung (JR 2005, S. 344). Die Einstellung der Bundesrichter spricht deshalb eher für die von Morgenstern vermutete rigidere und punitivere Entwicklung in der Rechtssprechung der Strafgerichte.

Für den Angeklagten soll es seit Prozessbeginn besonders wichtig gewesen sein, dass er nicht wegen Mordes verurteilt werde (so »die tageszei- tung « auf der Titelseite v. 31. 1., 1.2.04). Sein Verteidiger hat den hoffnungslosen Versuch unternommen die Tat als Tötung auf Verlangen umzudeuten. Beim Verfolgen dieses Prozesses drängt sich jedoch die Vermutung auf, dass es keinen juristischen Mittelweg geben kann. Es kollidieren nicht nur in der Bevölkerung, sondern auch in der Fachwelt zwei unvereinbare Positionen. Für die einen ist die Tat des Kannibalen kein Mord, weil der Angeklagte niemals die Schwelle zum schwersten Unrecht übertreten habe. Die anderen stellen darauf $a b$, dass es eine abscheuliche Tat gewesen sei. Mit Hilfe der genannten Positionen soll das moralische Dilemma des »Kannibalenfalls « und das Ringen um den Mordtatbestand noch einmal kurz verdeutlicht werden.

Die Subsumtion des vom LG Kassel ermittelten Sachverhalts unter die Tötungstatbestände reicht von Tötung auf Verlangen gem. § 216 StGB, was eine sehr niedrige Freiheitsstrafe, sogar eine Bewährungsstrafe ermöglichen würde (sechs Monaten bis zu fünf Jahren), bis hin zur lebenslangen Freiheitsstrafe wegen Mordes, ggf. sogar mit festgestellter besonderer Schwere der Schuld. Es steht die prozesstaktische Forderung der Verteidigung einer offenkundig polemischen Version der Staatsanwaltschaft gegenüber. Das Bemerkenswerte an diesem Prozess ist, dass große Einigkeit darüber besteht, dass dem Angeklagten weder Rechtfertigungs- noch Schuldausschließungsgründe zugute gehalten werden können. Mit Hilfe von Sachverständigen sind alle Zweifel über die Fähigkeit des Angeklagten, sein Unrecht zur Tatzeit zu erkennen und auch danach zu handeln, ausgeräumt worden (nicht ganz überzeugt von der Schuldfähigkeit ist lediglich Schiemann, NJW 2005, S. $2351 \mathrm{f}$.). Der Kannibalenprozess gibt somit keinen Raum für die üblichen Taktiken zur Umgehung der lebenslangen Freiheitsstrafe über subjektive Faktoren.

Die erste Frage, die sich stellt, ist die Bewertung der Motive des Angeklagten, der die Tötung eines anderen Menschen nur unter der Bedingung der Freiwilligkeit angestrebt hatte. Das spätere Opfer 
hingegen wurde von der Vorstellung angetrieben, erst durch eine Penisamputation während des Geschlechtsaktes den sexuellen Höhepunkt zu erreichen. Das Hauptproblem des Falles ist die Feststellung des Landgerichts Kassel, dass das Opfer diesen Moment als finales Erlebnis eingestuft hat, und nur deshalb bereit gewesen war, sein Leben zugunsten der kannibalistischen sexuellen Phantasie des Angeklagten zu beenden. Alle beteiligten Gerichte sind daher zu dem Ergebnis gekommen, dass der Angeklagte auch unabhängig von der Einwilligung zur Tötung entschlossen war, die Einwilligung des Opfers für den Täter also nicht handlungsleitend war, sondern nur die Tat erleichtert hat (zustimmend Schiemann, NJW 2005, S. 2351; nur im Ergebnis zustimmend Otto, JZ 2005, S. 800. Otto stellt darauf ab, dass der Angeklagte zwar durchaus von dem Opfer zur Tötung bestimmt wurde, das Verlangen des Opfers nach einer Tötung aber nicht ernsthaft sei, sondern nur Ausdruck der Vertragserfüllung. Er sieht aufgrund der krankhaften seelischen Störung des Opfers keine Möglichkeit, von einer freien, uneingeschränkten Willensbetätigung auszugehen.). Eine Privilegierung des Angeklagten wegen einer Tötung auf Verlangen nach § 216 StGB scheidet danach aus. Kudlich hingegen rückt die Opferautonomie in den Vordergrund. Für ihn begründet vor allem die Ablehnung des Opfers, zu seiner Rettung nach der Penisamputation einen Notarzt zu rufen, den Angeklagten zu privilegieren und den Rückgriff auf den Mordtatbestand dogmatisch zu sperren (JR 2005, S. 342).

Die Bewertung der Einwilligung des Opfers nicht nur in seine Tötung, sondern auch in die weitere Verwendung seines Körpers für die Umsetzung der Phantasien des Angeklagten, findet sich erneut bei der Frage, ob das Mordmerkmal der Ermöglichungsabsicht einschlägig ist. In Betracht kommt der Tatbestand der Störung der Totenruhe (§ 168 StGB), nämlich die Verübung von beschimpfenden Unfug mit der Leiche. Das Landgericht Kassel hat den beschimpfenden Charakter der Tat abgelehnt (ebenso Kudlich, JR 2005, S. 344). Der BGH sieht unter Rückgriff auf die Menschenwürde jedoch keine Möglichkeit des Opfers, in die spätere objektive Verunglimpfung seines toten Körpers, wie das Schlachten eine darstelle, einzuwilligen (zustimmend im Ergebnis Otto, JZ 2005, S. 800; zweifelnd Schiemann, NJW 2005, S. 2351).

Die zweite am Sachverhalt orientierte Aufgabe der Landgerichte war es zu klären, welchen Einfluss die sexuellen Phantasien des Angeklagten in dem konkreten Tatgeschehen hatten. Das Landgericht Kassel stellte fest, dass zentraler Bezugspunkt der sexuellen Erregung des Angeklagten das Aufschlitzen des Bauchraumes eines Mannes und das anschließende Ausweiden sei. In der Regel erlangte der Angeklagte seinen sexuellen Höhepunkt bereits vor der Vorstellung, das Menschenfleisch zu verspeisen. Die Ermitt- lungen ergaben, dass der Angeklagte bei der Tötung des Opfers keine sexuellen Erregungen verspürte, sich jedoch drei Tage später nach dem Verzehr des Fleisches die Videoaufzeichnung der Schlachtung ansah und dabei masturbierte. Der Bundesgerichtshof bemängelt, dass das Landgericht ungenügend aufgeklärt habe, ob das Video für den Zweck der Selbstbefriedigung hergestellt sei. Das Mordmerkmal »zur Befriedigung des Geschlechtstriebs « liege auch dann vor, wenn der Täter diese Befriedigung erst bei der späteren Betrachtung der Bild-Ton-Aufzeichnung (Video) vom Tötungsakt und dem Umgang mit der Leiche finden wolle (so der erste Leitsatz der BGHEntscheidung, NJW 2005, S. 1876). Die Ansichten zu dieser stark am Subjektiven orientierten Auslegung des Mordmerkmals gehen auseinander. Schiemann sieht gar keine Notwendigkeit für die von ihr abgelehnte neue Fallgruppe, dafür aber eine Parallele zu der Fallgruppe »Nekrophilie«. Sie stellt auf den sexuellen Bezug des auf die Tötung folgenden Ausweidens ab (NJW 2005, S. 2350). Otto mahnt den unmittelbaren Zusammenhang zwischen der Tötungshandlung und der Befriedigung des Geschlechtstriebes an (JZ 2005, S. 799). Das Töten des Opfers möchte er eher mit der Beseitigung eines Hindernisses vergleichen, für die der unmittelbare Zusammenhang verneint wird. Dieses Ergebnis möchte er aber damit korrigieren, dass aus seiner Sicht die Voraussetzungen des Mordmerkmals der niedrigen Beweggründe vorliegen. Kudlich schließlich stellt wieder auf den Fall der Nekrophilie ab und stuft gerade deshalb die Ausdehnung des Mordmerkmals durch den BGH auf die Videoaufzeichnung als konsequent sein (JR 2005, S. 343 f.).

Die Diskussion der Mordmerkmale macht die Vielschichtigkeit des Kannibalenprozesses deutlich. Zusammenfassend lässt sich sagen, dass die Kommentatoren des BGH-Urteils auf unterschiedlichen Wegen die Vorraussetzungen des Mordtatbestandes bejahen. Kudlich spricht sich darüber hinaus für das Vorliegen der Voraussetzungen der Privilegierung aus und vermeidet damit den Durchgriff auf den Mordtatbestand und das Eingreifen der lebenslangen Freiheitsstrafe. Aber wie hat schließlich das Landgericht Frankfurt a.M. entschieden? Der Angeklagte wurde gemäß den Vorstellungen des BGH wegen Mordes zu lebenslanger Freiheitsstrafe verurteilt: Ermöglichen der Störung der Totenruhe (Kannibalismus) und Befriedigung des Geschlechtstriebes (die Erinnerung an den Schlachtvorgang als sexuelle Stimulierung). Die Einwilligung des Opfers hat der Vorsitzende in seiner Urteilsbegründung mehrfach berücksichtigt. Unerheblich sei dieser Umstand für die Privilegierung, da der Angeklagte aus eigensüchtigen Motiven gehandelt habe. Entlastend wirkt die Einwilligung insofern, als dass damit die Feststellung der Schwere der Schuld abgelehnt wurde. Der Vorsitzende führte ebenfalls aus, dass die Tat nicht auf tiefster sittlicher Stufe anzusiedeln sei, da die Einwilligung des Opfers zu berücksichtigen sei. Andere niedrige Beweggründe erkannte das Gericht deshalb nicht (vgl. Holzhaider, SZ v. 10. 5. 06, S. 12).

Wie kann etwas nicht auf tiefster sittlicher Stufe stehen und trotzdem ein Mord sein? Das Bundesverfassungsgericht fordert, dass gewährleistet werden muss, dass die lebenslange Freiheitsstrafe auf Fälle von besonders verwerflichem Charakter beschränkt bleibe und dass diese Bestrafung des Täters im Verhältnis zur Schwere und zum Schuldgehalt seiner Tat angemessen sei (BVerfGE 45, S. 187; NJW 1977, S. 1525). Weder die wissenschaftliche Literatur noch die Rechtsprechung haben den Kannibalenfall zum Anlass genommen, trotz der rechtlich sehr uneinheitlichen Bewertung eine Gesamtabwägung vorzunehmen und auf diese zulässige und von der Rechtsprechung anerkannte Weise die absolute Strafandrohung zu mildern. Bei der Entscheidung des Landgerichtes Frankfurt a. M. wird deshalb mehr als deutlich, dass das Schwert der lebenslangen Freiheitsstrafe an Schärfe verloren hat. Den Platz der absoluten Strafandrohung scheint nun das Institut der » besonderen Schwere der Schuld « einzunehmen, verschärft durch die Möglichkeit lebenslange Freiheitsstrafe und Sicherungsverwahrung zu verbinden. So heißt es bei Holzhaider: »Lebenslang für den Kannibalen für Rothenburg, aber immerhin die Chance, nach 15 Jahren auf Bewährung entlassen zu werden « (SZ v. 10.5.06, S. 12). Greift man also noch einmal die Frage auf, wie ernst die Lage der lebenslangen Freiheitsstrafe sei, und lässt die Argumente aus dem Heft NK 2/2004 passieren, so scheint die Lage sehr ernst zu sein. Es bleibt daher abzuwarten, ob das Landgericht Frankfurt a. M. ein revisionsfestes Urteil verfassen wird, oder ob der BGH noch einmal die Gelegenheit zur Stellungsnahme bekommt und ergreift. Eingelegt ist die Revision, aber die Begründung kennen wir noch nicht.

Die Verfasserin ist Rechtsreferendarin im Landgerichtsbezirk Flensburg, Schleswig-Holstein und Doktorandin am Institut für Sanktionenrecht und Kriminologie der CAU zu Kiel. 